\title{
Myeloid Leukosis (J-virus) - An International Broiler Industry Concern ${ }^{1}$
}

\author{
Gary D. Butcher, DVM, Ph.D., and Richard D. Miles, Ph.D²
}

Myeloid leukosis (ML) or

myelocytomatosis/myeloblastosis is caused by a retrovirus in the Avian Leukosis/Sarcoma Group of Retroviruses (ALV). This ALV subgroup J virus (ALV-J), which is identified as the etiologic agent for ML, has caused substantial losses in heavy breeders in many areas of the world. The ALV-J was first isolated from heavy breeders and characterized in the United Kingdom in 1989. Investigators have demonstrated that the subgroup $\mathbf{J}$ virus appears to be a recombinant of subgroup $\mathrm{E}$ (endogenous) and an exogenous avian leukosis virus subgroup. In subgroup J virus isolates studied to date, antigenic and molecular diversity has been recognized between viruses from the USA and Europe. The $\mathrm{J}$ subtype virus appears to be genetically unstable, suggesting that the potential for the appearance of new variations within the $\mathrm{J}$ subtype and new subtypes are possible. The ALVs do not cause disease in man or other mammals.

The ALVs are divided into ten subgroups based on antigenic characteristics of their envelope (env) glycoproteins, host range, molecular characteristics, and other criteria (subgroups A through J). The viruses are also classified as being either exogenous or endogenous. Exogenous ALVs have the ability to infect birds by horizontal and congenital means and are not permanently incorporated into the host genome. Infection involves insertion of viral genetic material into the genome of the target cell, resulting in production of virus copies or cell transformation. Endogenous ALVs are permanently incorporated into the host cellular genome, do not produce virus copies, and are transmitted to progeny as the infected cells are passed to the offspring, i.e. genetic transmission. Subgroups A and B are the common exogenous field viruses associated with disease in Leghorn type chickens. Subgroups C and D are exogenous and are rarely reported in the field in chickens. Subgroup E includes the endogenous ALV present in practically all chickens. Subgroups F, G, and $\mathrm{H}$ are endogenous and found in the pheasant, and subgroup I is endogenous and is found in quail.

The ALVs responsible for lymphoid leukosis in leghorn chickens, subgroups A and B, can be transmitted both congenitally and horizontally. These exogenous viruses transform B-lymphocytes by integrating their reverse transcripted DNA provirus into the chicken's genome and then activating the cellular onc gene, resulting in the proliferation of the

1. This document is VM131, one of a series of the Veterinary Medicine-Large Animal Clinical Sciences Department, Florida Cooperative Extension Service, Institute of Food and Agricultural Sciences, University of Florida. Original publication date May 1, 2002. Visit the EDIS Web Site at http://edis.ifas.ufl.edu.

2. Gary D. Butcher, DVM, Ph.D., Diplomate, American College of Poultry Veterinarians, University of Florida College of Veterinary Medicine, Gainesville, FL., Richard D. Miles, Ph.D., Professor, Poultry Nutritionist, Department of Dairy and Poultry Sciences 
transformed cells. The J subtype ALV has only recently been identified in heavy breeders and has been shown to be widespread. This ML virus acts in a similar manner to subtypes $\mathrm{A}$ and $\mathrm{B}$, except that the primary target cell is the myelomonocytic series (bone marrow), with only a low tropism for B-lymphocytes. The chicken's genome also contains endogenous loci (endogenous ALV-like). These loci have little or no oncogenic potential. These are believed to have been derived from exogenous viruses that have become incorporated into the chicken's genome early in the evolutionary process. They are now passed from generation to generation in a simple Mendelian pattern.

The ALV-J subytpe contains 3 important genes from a diagnostic and/or taxonomic perspective. The gag gene is the ALV group-specific gene and its product is detected by commercially available antigen capture kits. The pol gene codes for the unique enzyme of retroviruses, reverse transcriptase and allows the ALV RNA to be converted to DNA for insertion into the host's genome. The env gene contains the genetic information for production of surface envelope proteins. One of these proteins, gp85, is unique for each of the known ALVs subgroups. This protein is associated with viral attachment and induces the production of subgroup-specific antibodies. Thus, a test system designed to identify gp85 of the $\mathrm{J}$ subtype will be able to identify the antibodies specific for the $\mathrm{J}$ subtype. However, bear in mind that the presence of env-specific antibodies will only indicate that prior exposure has occurred, not that disease is present or will occur in the future or that virus shed is occurring.

Evidence to date suggests that all strains of heavy breeders are susceptible, to a degree, to ML. Commercial leghorn chickens are also susceptible to infection, but appear to be resistant to tumor development. Myeloid-leukosis-infected flocks of heavy breeder may develop tumors involving the myelocyte cell line. Production problems in affected flocks include decreased fertility of hatching eggs due to male depletion or decreased number of eggs produced associated with female depletion. Shedding of virus and tumor expression are at a high level during the onset of egg production and may continue at a lower level for the life of the flock. Incidence of tumors is high in chickens infected with ALV-J by the congenital route. Horizontal transmission from fomites or infected chickens results in a lower incidence of clinical disease.

The differing severity of clinical disease associated with horizontal versus congenital transmission of ALV-J is explained by the phenomenon of immunotolerance. When the virus is congenitally transmitted or the chicks are horizontally infected at a very young age, the embryos and chicks may be immune tolerant to the infecting virus. These chicks will be viremic, but will not develop neutralizing antibodies. Thus these chickens will shed the virus congenitally and horizontally and will likely develop clinical disease with tumors. Chicks infected horizontally usually develop a transient viremia and then develop neutralizing antibodies. Only a small percentage of chickens in this category shed virus and the incidence of clinical disease is minimal. The resistance to tumor development occurs rapidly in horizontally infected chickens after the first weeks of life. However, environmental and management factors may delay the onset of this resistance. There are four possible categories of chickens to consider in suspect populations: 1) no viremia/no antibody/no shedding; 2) viremia/no antibody/shedding; 3) no viremia/with antibody/no shedding; and 4) few with viremia/with antibody/low-level shedding.

On necropsy of ML-caused mortality, tumors are found in the majority of breeders. Tumors are primarily myelocytomas and myeloblastomas. ML-related mortality increases, usually beginning at approximately 17 weeks of age. A number of variables affect age of disease onset and mortality pattern. These include genetics of the chicken, age of infection, immunosuppressive diseases, concurrent diseases, strain of ALV-J involved, and management and environmental factors.

Marek's disease challenge in susceptible flocks or challenge with highly virulent Marek's disease viruses is associated with immunosupression and an increased incidence of ML. Improved Marek's vaccination practices coupled with improved sanitation have resulted in reduced losses from myeloid leukosis. Improved control of infectious bursal disease, reovirus infections and chicken 
anemia agent, and reduced consumption of mycotoxins should also result in a reduced incidence of clinical ML. These diseases are associated with immunosupression and result in increased susceptibility to ML and greater severity of ML infection.

In most cases, horizontal infections with ALV-J in breeders do not result in clinical disease. That is, in the absence of tumor development, the effects of ALV-J infection in flocks is minimal. However, tumor development will result in progressive disease, resulting in culling or death.

The impact of ML on broilers is poorly understood. Broilers from affected breeder flocks typically have reduced performance. It is reported that broiler flocks originating from breeders with high levels of ML virus shed will be poorly uniform and pale in color with poor feathering and increased late mortality. Whether this loss of broiler performance is due to viral infection and resulting immunosuppression or simply due to the hens being debilitated and producing weak chicks is not clear.

ML virus, behaving as an exogenous leukosis virus, is shed both congenitally and horizontally by infected breeders. Horizontal transmission is through close contact with infected chickens or fomites and is very efficient during the first weeks of life. Subgroup $\mathrm{J}$ virus appears to spread more rapidly in chicks than other subgroups of ALV during the initial weeks post hatch. Congenital transmission occurs in hens, which shed ML virus from oviduct secretions to the egg albumen and then chicken embryo. ML-infected breeders have been shown to shed virus to progeny at a high level. In males, the ML virus is present throughout the reproductive tract and can be detected in the semen. There is no evidence that baby chicks become infected through contaminated semen, however, males may infect the hen venereally.

ALV-J is present in heavy type breeders throughout the world. Diagnoses in most cases have been based on clinical history and on finding characteristic gross and microscopic lesions. Mortality associated with ALV-J will normally increase near onset of egg production, but may also occur at younger and older ages. Clinically affected birds are weak, recumbent, and experience weight loss. Gross lesions include diffuse to nodular, soft and friable, white-yellow tumors, typically associated with bone, especially the keel, ribs at the costochondral junction, vertebral column, and pelvis. Tumors are also found in the soft tissues including the liver, spleen, kidney, lung, peripheral nerve, and others. Histologically, the infiltrates consist of solid masses of uniformly differentiated mature myeolocytes with abundant eosinophilic cytoplasmic granules. Confirmation of ML should be confirmed through isolation of the virus and identification.

A specific enzyme labelled immunosorbent assay (ELISA) test to identify ALV-J has been developed. ALV-J has been found in blood serum, cloacal swabs, and from tumors themselves.

Management practices recommended to reduce losses associated with ML include 1) separate rearing of males and females to reduce potential of horizontal transmission. Ideally, they should be raised separately until transfer to the breeder farm. 2) Improve biosecurity for all types of traffic between male and female houses. For example, ML can be transmitted horizontally by people, equipment, and vehicles moving between houses. Contaminated needles, vaccination equipment, and beak trimming equipment should be decontaminated between houses. 3) Closely follow nutritional and body weight recommendations to reduce stress. 4) Ensure adequate feeder/water space to reduce competition and stress. 5) Maintain appropriate male/female ratios. Another practice being discussed by breeder managers to control ML is associated with the breeder Marek's disease vaccination program. Although there is no scientific data to support this practice in heavy breeders, many managers are removing the SB-1 Marek's vaccine from their programs and using the Rispens and HVT combination. It has been documented in the past that use of serotype 2 Marek's disease vaccines in specific strains of Leghorns was associated with an increased susceptibility to lymphoid leukosis. There is no evidence, at this time, to suggest that a similar relationship exists between administering SB-1 and having an increased susceptibility to ALV-J in heavy breeders. Time will tell if this practice is in reality counterproductive. 